\title{
XXIII ASTIN COLLOQUIUM, STOCKHOLM, 1991
}

The 23rd ASTIN Colloquium was held from 30th June to 4th July 1991 in Stockholm ("beauty on water"). Over 200 actuaries from more than 20 countries attended. The Colloquium began on Sunday, 30th June, with the reception in Berns Congress Centre. The working sessions were started off on Monday morning with a presentation by Prof. Ragnar Norberg (University of Copenhagen) on "A Continuous Time Approach to the Prediction of the Total Outstanding Claims of a Non-Life Insurance Business - a Strategy for Solvency Control Based on the Break-Up Point of View". The rest of the morning and the afternoon were devoted to working sessions on topic number 3 "Modern Statistical Techniques". Bengt von Bahr and Arne Sandström opened these sessions by a brief survey of the papers on this topic. Then each author had 10 minutes to present his paper in greater detail. The remaining time was open for discussion, which was very animated. In the evening, the Colloquium participants were invited to a buffet dinner and a tour of the Stockholm City Hall, in which the annual Nobel Prize awards banquet is held.

An all-day excursion to the Gripsholm Castle was on the program for Tuesday, the very day on which midsummer weather set in Sweden. In two ships, the participants were taken directly from the centre of Stockholm to the outlet of Lake Mälaren. After having passed through Stockholm the journey continued through a majestic forest and lake countryside to the Gripsholm Castle, situated directly on the lake some $60 \mathrm{~km}$ inland. Lunch had been served on the ships, so that the afternoon was left open for a tour of the numerous rooms of the 16th Century Castle with its impressive portrait gallery of important figures from Swedish history. The lovely location of the Castle was also an invitation to linger on, which only made it all the more difficult to get onto the waiting busses for the trip back.

Wednesday was again devoted exclusively to working sessions. These were introduced with a presentation by Karl-Olof Hammarkvist, Managing Director of a large Swedish direct insurance and reinsurance company, concerning a "Professional View on Reinsurance". The subsequent working sessions dealt with Colloquium topics number 2 ("High Tech Reinsurance") and 1 ("The Use of Financial Theory in Insurance"). The "rapporteurs" were Björn Ajne, Malcolm Campbell and Björn Palmgren. The afternoon was concluded with the General Meeting, for which a special election to the ASTIN Committee was scheduled in addition to regular items on the agenda. Thomas Mack (Germany) and Ermanno Pitacco (Italy) were elected to the ASTIN Committee which now consists of 13 members. In the evening, the traditional Colloquium dinner was served on the "Operaterrassen" with a wonderful view of the old part of the town and the Royal Castle of Stockholm. 
The topic of the last working session on Thursday morning was "Speakers" Corner". Here papers were presented and discussed which had only been submitted shortly before the beginning of the Colloquium and could therefore not be included in the Colloquium publication, as it had already been sent to the participants in advance.

So much for the formal "contours" of the Colloquium, which had been extremely well organized by the Swedish Society of Actuaries, headed by Alf Guldberg. The members of the organizing committee (Sven Astrand, Hans Ekhult, Bengt Langhed, Peter Lindström, Gunilla Lisén, Harry Wide) and the scientific committee (Björn Ajne, Bengt von Bahr, Anders Blommé, Malcolm Campbell, Björn Palmgren, Arne Sandström) deserve special thanks.

The following is a brief summary of the contents of all papers presented. These summaries are of course influenced by the capabilities of the authors of this report and may therefore be somewhat biased. The order of the summaries is also the order in which they were presented at the Colloquium.

Thomas Mack, Klaus-Peter Mangold

\section{LIST AND SUMMARY OF PAPERS}

\section{Topic 3: Modern Statistical Techniques}

Björn Ajne and Arne SAndström: New Standard Regulations Regarding Allocation of the Safety Reserve in Sweden.

The authors explain in detail how the current Swedish safety reserve regulation came into being. For every line of business, there is a maximum tax-free value admitted for the safety reserve which consists of a percentage of premium income and a percentage of the claims reserve (due to the settlement risk), each of these being for own account. The percentages had been fixed in such a way that these total approximately 4.5 times the standard deviation of the loss ratio. This value was established on the basis of confidence interval considerations (99\% security) calculated from statistics. Allowance was also made for the positive correlation of loss ratios for two consecutive years observed. The paper contains many illustrative graphical displays.

John BorregaArd, Chresten Dengsoe, Joakim Hertig, Niels Jespersen and Christian Roholte LARSEN: Equalization Reserves: Reflections by a Danish Working Party.

Since there is no fixed regulation in Denmark governing the level of equalization reserves, the Danish Association of Actuaries formed a relevant working party to deal with this question. In the present paper, members of this party outline the method of balanced limits for setting up an upper limit $u$ and a lower limit $l$ for the equalization reserve. If the annual claims amount $x$ is less than $l$, the difference $l-x$ is transferred to the equalization reserve. If $x$ is greater than $u$, then $x-u$ is transferred from the reserve. On the basis of three sample calculations for Windstorm, Fire and Motor insurance, the authors demonstrate this method on the basis of theoretical distributions with realistic 
parameters. Furthermore, they propose setting the reserve at a level at which the probability of the reserve being completely exhausted within 5 or 10 years is below a given value (for example $15 \%$ ). The levels which result from this for the examples cited are also shown.

Arne Sandström: On Moment Corrections when Data are Grouped into Non-Equidistanced Intervals.

For equidistantly grouped data Sheppard's correction for calculating the moments can be applied. The author proposes formulae for the nonequidistant case, which use only group frequencies and lengths and mid-points of intervals. For equidistantly grouped data, these formulae lead to higher values than Sheppard's formula. If the mean value per interval is also known, upper and lower limits can also be given for the higher moments. The author indicates that it is thus possible to demonstrate a superiority of this formula over Sheppard's correction.

Rolf Larsson and Erik Hevreng: On the Estimation of the Time Development of the Risk Premium in Non-Life Insurance.

The authors fit mathematical distribution models to empirical claims data (number of policies, amount of every individual claim for 11 years and 4 different lines of Property business). For the inflation-adjusted claim amount, they assume a lognormal distribution with a scale parameter depending linearly on the year of observation. A Poisson distribution is assumed for the claims number per year, whose mean value is modelled as a product of the number of policies and a logarithmic linear yearly trend. In numerous tables, the authors give a year-by-year comparison between the observed value and the fitted value (for claims number, claims amount and risk premium per policy) and place special emphasis on the examination of the significance of modelled trends. Since the authors originally only had access to the amounts of the individual claims grouped by classes of claims amount, they originally estimated the parameters by means of the $E M$-algorithm briefly described in an appendix. The resulting estimators are also included in the tables. The authors therefore regard the comparison of the results of both methods as being one of the main issues of their paper.

\section{Erik Elvers: A Note on the Generalized Poisson Distribution.}

The author fits the Generalized Poisson Distribution introduced by Consul to the data observed in an Automobile Third Party Liability insurance portfolio. These data do not represent the number of accidents, but the number of casualties (injuries or deaths) per accident (over a period of several years and for several classes of motor vehicles). In almost every case, the chi-square test rejects the distribution hypothesis.

Jean Lemaire: Negative Binomial or Poisson-Inverse Gaussian?

On the basis of six data sets not related to insurance, the author compares the fit of the Poisson, the Negative Binomial and the mixed Poisson-Inverse Gaussian distributions. The parameters are estimated with both the maximum 
likelihood method and the method of moments. The best fits were obtained with the Negative Binomial distribution.

Ermanno Pitacco: An Inference Model for Risks with Variable Claim Frequency Rate.

The author generalizes the classical Poisson-Gamma model for the purpose of experience rating in Sickness insurance. For an insured life aged $y$ and a policy term of several years, for the conditional claims number variable $X(y+h)$ a Poisson distribution with parameter $\theta t(y+h)$ is assumed, where $t(y)<t(y+1)<t(y+2)<\ldots$ If the risk parameter $\theta$ follows a Gamma distribution, one can procede with a calculation analogous to the classical case. From this, the author analyses the properties of an experience rating system on the assumption that the individual claims amounts are independent of both the claims number and the parameter $\theta$.

Lourdes Centeno and João Manuel Andrade E Silva: Generalized Linear Models under Constraints.

After giving a brief description of the generalized linear models and the relevant estimation algorithm, the authors address the problem that in some applications the coefficients of the independent variables should satisfy certain linear constraints. In the authors' example which is based on Portuguese Automobile Third Party Liability insurance statistics, for example, this always occurs where no distinction is to be made between two variables or if one wishes to establish a certain fixed linear relationship. The authors offer two possible solutions: either reformulating the model or changing the algorithm. In the authors' opinion, the latter is easier as long as there are several step-by-step changes in the constraints, as is often the case in practice.

Pierre Petauton: Une Estimation Naturelle des Paramètres Structuraux dans les Modèles de Crédibilité.

The credibility estimator

$$
\hat{X}_{i}=z_{i} \bar{X}_{i+}+\left(1-z_{i}\right) m
$$

in the models of Bühlmann or Bühlmann and Straub includes the unknown parameters $z_{i}$ and $m$, which must be estimated from the data $X_{i j}$. To this end, the author proposes a new approach. For a fixed year $j$, he eliminates the relevant observations $X_{i j}, i=1,2, \ldots$, and applies the above credibility formula to the remaining data, which means,

$$
\hat{X}_{i}(j)=z_{i} \bar{X}_{i+}(j)+\left(1-z_{i}\right) m
$$

where $\bar{X}_{i+}(j)$ is the mean of the data without year $j$. This can be applied to each year $j$ of every risk $i$. The new approach is then to choose the parameters $z_{i}$ and $m$ in such a way that they minimize the weighted quadratic deviation

$$
\sum_{i, j} g_{i j}\left(X_{i j}-\hat{X}_{\mathrm{i}}(j)\right)^{2}
$$


where the weights $g_{i j}$ should be in inverse proportion to $\operatorname{Var}\left(X_{i j}\right)$. This idea can be easily carried out in the simple Bühlmann model and, with a small exception, produces the usual estimators. In the Bühlmann-Straub model, the author must alter the expression to be minimized in order to arrive at explicit solutions.

\section{Erhard KREMER: Large Claims in Credibility.}

The author implements ideas from robust statistics in the credibility theory in order to cope with the disturbing influence of outliers (large claims or risks) which disrupt the homogeneity assumption. To this end he proposes replacing the individual loss experience contained in the credibility formula with robust estimators. This is done by applying so-called $M$ - or $L$-estimators. For three examples, the so-called Huber credibility $M$-estimator, the trimmed and the winsorized credibility $L$-estimators he demonstrates robustness, which is not a property of the classical credibility estimator. The author does not directly truncate individual claims, but gives large claims less weight in the estimation or does not even take them into consideration at all. In the same manner, for estimating the structural parameters, he does not use the observations themselves, but rather robust statistics of the observations in order to obtain a kind of robust empirical credibility estimator. In the last chapter the author extends his approach to the regression credibility model as well.

\section{Arne Eyland: Classification of Passenger Cars in a Multiplicative Rating} Model using Recursive Credibility Estimation.

The author develops an evolutionary regression credibility model, whose basic features are derived from a paper of SUNDT (1987). In this model, the non-observable parameter which characterizes each single risk may vary over time (time-heterogeneous model) and its risk premium is regressively dependent on observable technical variables. The multiplicative tariff used by a Norwegian insurance company, in which the tariff variable "car model" is determined by the technical parameters engine power, price and weight, serves as an example. The problem of having to invert the covariance matrix of the observations for determining the credibility estimator is reduced in its dimension by using linear sufficient statistics. Furthermore, the selection of a special recursive covariance structure in the model makes it possible to calculate the credibility estimator recursively. The author also treats the special case of risk parameters that are constant in time (time-homogeneous model) which he believes to be less realistic. In a third, so-called time-heterogeneous model for two portfolios, the author describes the situation in which the overall portfolio can be broken down into two sub-portfolios, each of these being timeheterogeneous and transition from one sub-portfolio to the other being possible. This would be useful in a case in which a technical variable ("price") is "suddenly" no longer observable (e.g. because a car model is no longer sold) and thus has to be subsumed under the unobservable risk parameter. The author purposely excludes the problem of estimating the (numerous) structural parameters and indicates that this will be investigated in a later publication. 


\section{Topic 2: High Tech Reinsurance}

William S. JEweLL: The Value of Information in Forecasting Excess Losses.

The author analyses parameter estimation for rating an excess-of-loss treaty. Usually, the reinsurer has at his disposal only information on losses exceeding a certain limit. The author explains the problem resulting from this limited knowledge for the case of a Poisson distributed number of losses with shifted Pareto distributed loss amounts. By means of many impressive graphs he shows that the likelihood function of the two distribution parameters (the Poisson parameter and the Pareto shift parameter - the shape parameter being treated as known) has a ridge which allows only a very uncertain parameter estimation, if any at all. This situation can only be improved if at least the number of all losses from the ground up is available. The paper also addresses questions like the direct estimation of the excess-of-loss fair premium.

Erhard Kremer: $A$ (New) Nonparametric Method for XL-Rating.

The author premises the fact that claims expectancy under an excess-of-loss reinsurance treaty can be represented as the product of the total claims expectancy of the insured portfolio and a tail probability

$$
\int_{P}^{\infty} g(x) d x
$$

above the priority $P$. In view of the infinite integration range, he proposes using the approximate procedure of GRAY and LEWIS (1971) for calculating this tail probability. This procedure uses the quotient of two determinants of the first $k$ derivatives of $g$ at the priority $P$. In order to avoid parametrically estimating the density $g$ with all its derivatives from the data, the author recommends using the kernel estimator. For this, it is necessary to choose a specific kernel function, for which the author gives a concrete recommendation.

Erhard KREMER: $A$ Note on XL-Rating in Earthquake Insurance.

Referring to a paper by MAKJANIC (1980), the author proposes use of the generalized exponential distribution for the magnitude frequency instead of the traditional exponential distribution. The author also offers a modification of Makjanic's parameter estimation procedure.

Patrik Dahl: Some Reflexions on Contingent Premium Payment Plans.

The author investigates reinstatement agreements in non-proportional reinsurance on the assumption that no partial claims in respect of the reinsurer's liability are possible and that both parties evaluate the treaty with an exponential utility function. Furthermore, the author makes allowance for the 
possibility that insurer and reinsurer may calculate with differing claims probabilities. Allowance is also to be made for the resulting cash flow as the premium of the reinstatement agreement depends on claims experience. In another part of the paper, the author argues quite generally for use of the Polya process as a model for the claims number.

\section{Marc-Henri AMSLER: Réassurance du Risque de Ruine.}

The author proposes for pension funds, for example, that a reinsurance treaty with a term of several years be applied, under which the reinsurer is only obliged to pay in the event of ruin and only for the amount of the deficit. Here the reinsurer's payment needs only to be a loan, since, in view of the positive security loading, the reinsured's contingency reserve will tend to become higher and higher. At expiry of the treaty, the reinsurer is obliged to pay if the reinsured's contingency reserve is lower than at the outset. The author calculates the resulting reinsurance premiums in two examples.

Thomas MACK : Claims Reserving: The Direct Method and its Refinement by a Lag-Distribution.

The author describes a simple procedure for claims reserving, which has some similarity to the chain ladder method. It is however also possible to show that the procedure represents the maximum likelihood estimator if a gamma distribution is assumed. The procedure can be used in a more realistic manner if it is only applied for the average claims amount per cell. In this case, the triangle of the number of payments must also be completed to make a square. To this end the author proposes to fit a (truncated) lag-distribution to the observed numbers of payments per year of occurrence. This proved to be more suitable than the chain ladder method in the practical example given by the author.

Gunnar Benktander: A Special Case of Variable Rates in Excess of Loss.

In order to make a variable premium with a minimum of $m$ and maximum of $M$ produce the same average result as a constant premium $E, m$ and $M$ must be fixed in a specific way. The author investigates this question for excess of loss reinsurance, basing his investigation on a Poisson-Pareto distribution model. On the basis of the simulations conducted by Christer Möller, he demonstrates that the "accordion rule" $m M=E^{2}$ closely approximates the simulated results. The author also raises the question as to whether there are distributions for which this rule applies exactly. A paper partially responding to this question was distributed by Björn Sundt.

\section{Mette RytgaARD: Variations on Typical Excess of Loss Covers.}

The author initially investigates an excess of loss cover which provides a reinstatement of the cover for an additional premium only. In this case, the calculation of the reinsurance premium becomes simpler if one directly examines the reinsurer's net payment after deduction of the claims-related additional premiums. This is also shown to be true for other agreements, such 
as, for the variable premium. Especially in the case of the variable premium however the net payment of the reinsurer might also be negative due to the multiplicative premium loading, for which reason the author proposes a form of the variable premium which avoids this disadvantage.

\section{Topic 1: The Use of Financial Theory in Insurance}

\section{David SANDERS: Risk Theory and Capital Allocation.}

The author uses the formulae of the classical ruin theory to derive a link between the premium loading, the variance of the total claims amount, the capital allocated to support the business and the probability of ruin under the assumption that the total claims amount has a normal distribution. Using the required rate of return on capital as a part of the premium loading he obtains a functional relationship between premium and capital which has a minimum point. The author analyses the minimum condition from the standpoint of its practical implications and gives some numerical examples.

\section{Speaker's Corner}

Siegfried Kuon, Michael Radtke and Axel Reich: The Right Way to Switch from the Individual Risk Model to the Collective One.

The overall loss $S_{\text {ind }}=X_{1}+\ldots+X_{n}$ in the individual model of $n$ independent and not necessarily identically distributed risks $X_{i} \geqslant 0$ is usually approximated by a collective model $S_{\text {coll }}=Z_{1}+\ldots+Z_{N}$ with a claims number variable $N$ and i.i.d. claims amounts $Z_{j}>0$. Here the distribution of the $Z_{j}$ is

$$
p\left(Z_{j} \leqslant x\right)=\sum_{i=1}^{n} q_{i} p\left(X_{i} \leqslant x \mid X_{i}>0\right) \mid \sum_{i=1}^{n} q_{i}
$$

with $q_{i}=p\left(X_{i}>0\right)$. If one chooses a Poisson distribution for $N$ so that $E\left(S_{\text {coll }}\right)=E\left(S_{\text {ind }}\right)$ applies, it is known that $\operatorname{Var}\left(S_{\text {coll }}\right)>\operatorname{Var}\left(S_{\text {ind }}\right)$. The authors show that this is also the case if one chooses the negative binomial distribution or the binomial distribution for $N$ (at least if not all $E\left(X_{i}\right)$ are equal).

Furthermore, it is shown that in the case of a growing portfolio, i.e. $n \rightarrow \infty$, $\operatorname{Var}\left(S_{\text {coll }}\right) / \operatorname{Var}\left(S_{\text {ind }}\right)$ does not converge toward 1 and that, in like manner, neither distribution functions nor percentile premiums converge toward one another either. Finally, it is shown that, on the one hand, by passing from $Z_{j}$ to $a Z_{j}$ with $a<1$, the variances can be made equal, but, on the other hand, that the relevant stop-loss net premiums will still differ from one another significantly.

\section{Björn PALMgren: Financial Risk in Insurance.}

The author gives a brief survey of a number of questions on the financial risk of an insurance company, particularly from the standpoint of the supervisory 
authority, e.g., "When is a mix of assets efficient or at least acceptable?", "How great is the influence of transaction costs?", etc. The author sees it as an important task of the supervisory authority to investigate the efficiency of capital investments and conduct sensitivity tests on company solvency.

Ermanno PITACCO: Selection and Experience Rating in Health Insurance.

This paper is a sequel to the paper by the same author discussed earlier under topic 3. The age-related parameters $t(y+h)$ mentioned in the earlier paper are lower under the influence of medical selection than without selection. This also influences the a posteriori expected value. The author gives a numerical example and also shows how a given selection assumption affects different experience rating system concepts.

\section{Erhard KREMER: The Total Claims Amount of Largest Claims Reinsurance Treaties Revisited.}

The author takes up the total claims amount of the reinsurer under the generalized largest claims reinsurance which he himself introduced in an earlier paper. The purpose of this paper is to provide a formula for the distribution density of the total claim where the individual claims are either exponentially or uniformly distributed. To this end, the author first investigates the special case in which exactly $n$ claims are incurred. The resulting expressions are not simple and must still be mixed with the occurrence probabilities.

Rob KaAs, Marleen Vanneste and Marc Goovaerts : Maximizing Compound Poisson Stop-Loss Premiums Numerically with Given Mean and Variance.

In contrast to earlier papers, the authors limit themselves here to total claims amounts with a compound Poisson distribution. Furthermore, they assume that the single claim amount is limited and arithmetic. They provide a numerical solution which is arrived at by means of the gradient method, which however shows that there are many local maxima. In addition to this, the maximum frequently deviates only very slightly from the value that is produced with a two-point distribution approximation for the single claim amount. The authors therefore feel that one should be satisfied with the procedure outlined in another paper by KAAS (1991) which uses two-point distributions.

Björn Sundt: On Some Extensions of Panjer's Class of Counting Distributions. The author generalizes the class $R_{1}$ of the claims number distributions with

$$
p_{n}=(a+b / n) p_{n-1}
$$

to class $R_{k}$ with the recursion property

$$
p_{n}=\sum_{i=1}^{k}\left(a_{i}+b_{i} / n\right) p_{n-i}
$$

Distributions from $R_{k}$ are a result of convoluting distributions from $R_{1}$. The author shows that the Panjer algorithm can also be translated to distributions 
of class $R_{k}$. A formula for convoluting any two distributions of class $R_{1}$, which is known to include the binomial, Poisson and negative binomial distributions, is a by-product of this.

Björn AJNE: A Note on the Additivity of Chain-Ladder Projections.

The author investigates the problem of when the separate application of the chain ladder method to 2 run-off triangles produces the same projections as the single application to the sum of both triangles and he outlines a necessary and sufficient condition for this. He also conjectures as to when separate application produces lower reserves.

\section{Jon Holtan: Bonus Made Easy.}

To begin with, the author points out a few weaknesses of the traditional bonus-malus systems, for example, that the amount of a claim has no influence. In order to get around these weaknesses, he proposes introduction of a relatively high deductible as a substitute for the bonus-malus system. With a high deductible, a claim would have to be financed in advance by the insurer. In order to find the optimum deductible amount and mode of loan repayment, he applies a special loss function. The author sees a potential practical difficulty in the fact that, with this solution, the credit risk of the insured might be a factor to be considered.

Heikki BonsDorfF: On the Convergence Rate of Markovian Bonus-Malus Systems.

Under certain conditions, transition probabilities of a Markovian bonusmalus system converge toward stable transition probabilities. For such convergent Markov chains, the expression "convergence rate" is well-defined and can be calculated with the help of the eigenvalues of the transition matrix. The author calculates this convergence rate for the Dutch, Swiss and Finnish bonus-malus system, the results of which show that the latter converges most quickly. 